\title{
ON INDUCED MORPHISMS OF MISLIN GENERA
}

\author{
Peter Hilton \\ Dedicated to my good friend Karl Gruenberg, in admiration and affection, \\ on the occasion of his $65^{\text {th }}$ birthday
}

\begin{abstract}
Let $N$ be a nilpotent group with torsion subgroup $T N$, and let $\alpha: T N \rightarrow \tilde{T}$ be a surjective homomorphism such that $\operatorname{ker} \alpha$ is normal in $N$. Then $\alpha$ determines a nilpotent group $\tilde{N}$ such that $T \tilde{N}=\tilde{T}$ and a function $\alpha_{*}$ from the Mislin genus of $N$ to that of $\tilde{N}$ if $N$ (and hence $\tilde{N}$ ) is finitely generated. The association $\alpha \mapsto \alpha_{*}$ satisfies the usual functorial conditions. Moreover $[N, N]$ is finite if and only if $[\tilde{N}, \tilde{N}]$ is finite and $\alpha_{*}$ is then a homomorphism of abelian groups. If $\tilde{N}$ belongs to the special class studied by Casacuberta and Hilton (Comm. in Alg. 19(7) (1991), 20512069), then $\alpha_{*}$ is surjective. The construction $\alpha_{*}$ thus enables us to prove that the genus of $N$ is non-trivial in many cases in which $N$ itself is not in the special class; and to establish non-cancellation phenomena relating to such groups $N$.
\end{abstract}

\section{Introduction}

Guido Mislin introduced and discussed in $[\mathbf{M}]$ the genus $\mathfrak{G}(N)$ of a finitely generated $(f g)$ nilpotent group $N$. This consists of isomorphism classes of $f g$ nilpotent groups $M$ such that

$$
M_{p} \cong N_{p}, \text { for all primes } p,
$$

where $M_{p}$ is the $p$-localization of $M$. By abuse we say that $M$ belongs to $\mathfrak{G}(N)$. It was early known that $\mathfrak{G}(N)$ is not trivial, but systematic methods of calculating the set $\mathfrak{G}(N)$ and representing its elements were lacking.

Mislin himself in $[\mathbf{M}]$, and together with the present author in $[\mathbf{H M}]$, described an abelian group structure which could be introduced into $\mathfrak{G}(N)$ if $N$ satisfied the condition that its commutator subgroup $[N, N]$ 
is finite; we call the class of such $f g$ nilpotent groups $\mathfrak{R}_{0}$; moreover, $\mathfrak{G}(N)$ is then finite. However, this still did not permit any kind of systematic calculation of $\mathfrak{G}(N)$. Calculations were done for specific groups in [H2]. Later, Casacuberta and Hilton $[\mathbf{C H}]$ introduced a class of nilpotent groups $\mathfrak{R}_{1} \subset \mathfrak{R}_{0}$, and calculated $\mathfrak{G}(N)$ for $N \in \mathfrak{R}_{1}$; they further showed how to modify $N$ to realize any given element in $\mathfrak{G}(N)$. The nature of the groups in $\Re_{1}$ was further analysed in [S], [HS1] -indeed, the class is very strongly restricted - and, in [S], [HS2], the calculation of the genus was extended from $N$ to $N^{k}$, the direct product of $k$ copies of $N$, provided $N \in \Re_{1}$. A key result in this work is that, for $N \in \mathfrak{R}_{1}$, $\mathfrak{G}(N)$ can only be non-trivial if $F N=N / T N$ is cyclic, where $T N$ is the torsion subgroup of $N$; recall that $F N$ is commutative for $N \in \Re_{0}$.

A significant difficulty in attempting to calculate $\mathfrak{G}(N)$ is that $\mathfrak{G}$ lacks any kind of functoriality. We endeavor in this paper to go some way towards remedying this defect. Thus we suppose given a $f g$ nilpotent group $N$ and a surjective homomorphism $\alpha: T N \rightarrow \tilde{T}$, for some finite group $\tilde{T}$ which is, of course, necessarily nilpotent. Given the supplementary condition that $\operatorname{ker} \alpha$ is normal in $N$, we construct a $f g$ nilpotent group $\tilde{N}$ such that $T \tilde{N}=\tilde{T}$ and a function $\alpha_{*}: \mathfrak{G}(N) \rightarrow \mathfrak{G}(\tilde{N})$. Moreover, $N \in \mathfrak{R}_{0}$ if and only if $\tilde{N} \in \mathfrak{R}_{0}$; and $\alpha_{*}$ is then a homomorphism. It is easy to see that $\alpha \mapsto \alpha_{*}$ satisfies the usual functoriality conditions. Further we show in Section 2 that if $\tilde{N} \in \Re_{1}$ then $\alpha_{*}$ is surjective; thus, in this case, considerable information is made available about $\mathfrak{G}(N)$, since we may calculate $\mathfrak{G}(\tilde{N})$.

A particular, and important, example of the construction is afforded by taking $\tilde{T}$ to be the abelianization of $T N$ with $\alpha$ the abelianizing homomorphism. To avoid triviality we take $F N$ cyclic. Then $\tilde{N}$ satisfies two of the three conditions for membership of $\mathfrak{R}_{1}$ (see below). Moreover, the third condition will be automatically satisfied if $\tilde{T}$ happens to be cyclic.

We also show in Section 2 that a non-cancellation result proved in [CH] for groups in $\mathfrak{R}_{1}$ extends to groups, which, in our sense above, lie over groups in $\Re_{1}$. That is, we obtain pairwise non-isomorphic groups $(L, M, \ldots)$ in $\mathfrak{G}(N)$ such that $L \times C \cong M \times C \cong \ldots \cong N \times C$, where $C$ is cyclic infinite.

In Section 3 we give a typical example of the application of the method, with explicit calculations.

For the convenience of the reader, we collect here the crucial facts about the class $\mathfrak{R}_{1}$. We assume $N \in \mathfrak{R}_{0}$ and refer to the extension

$$
T N \mapsto N \rightarrow F N
$$


Then $N \in \Re_{1}$ if

(i) $T N$ is commutative;

(ii) (0.2) is a split extension for an action $\omega: F N \rightarrow \operatorname{Aut} T N$;

(iii) $\omega(F N)$ lies in the center of Aut $T N$.

We then note that, in the presence of (i), condition (iii) is equivalent to

(iii)' for each $\xi \in F N$, there exists a positive integer $u$ such that $\xi \cdot a=$ $u a$, for all $a \in T N$.

To avoid a trivial genus, we assume $F N$ cyclic, say, $F N=\langle\xi\rangle$. Let $t$ be the order of $\omega(\xi)$ in Aut $T N$. Then [CH], if $N \in \Re_{1}$,

$$
\mathfrak{G}(N) \cong(\mathbb{Z} / t)^{*} /\{ \pm 1\} .
$$

Moreover, if $[m] \in(\mathbb{Z} / t)^{*} /\{ \pm 1\}$, where $m$ is prime to $t$, we may choose the isomorphism (0.3) so that the group $N_{m}$ corresponding to $m$ is obtained from $N$ by introducing a new action $\omega_{m}$ of $F N$ on $T N$, defined by

$$
\omega_{m}(\xi)=\omega\left(\xi^{m}\right) .
$$

A final remark pertains to the general construction in Section 1. There is no need to insist that $N$ be $f g$ to carry out the construction. Thus Theorem 1.1 may be extended to yield a function $\alpha_{*}$ from the extended genus of $N$ to the extended genus of $\tilde{N}$ (see [H3]).

\section{The construction}

Let $N \in \Re_{f g} \subset \Re$; that is, $N$ is a $f g$ nilpotent group. There is then a canonical exact sequence

(1.1) $T N \stackrel{i}{\mapsto} N \stackrel{\pi}{\rightarrow} F N, T N=$ torsion subgroup of $N$,

$$
F N=\text { torsionfree quotient }
$$

Now let $\alpha: T N \rightarrow \tilde{T}$ be a surjection, so that $\tilde{T}$ is a finite nilpotent group. Assume that $\operatorname{ker} \alpha$ is normal in $N$; call this condition $K$. Then we know [H1] that we may embed (1.1) in a map of exact sequences

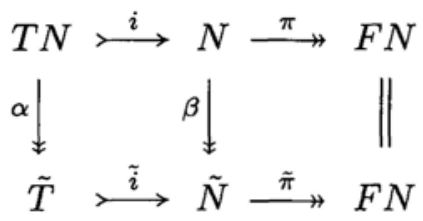


with $\tilde{N} \in \Re_{f g}$. Moreover, the LHS of (1.2) is a push-out in the category of groups; and, obviously, $F \tilde{N}=F N, T \tilde{N}=\tilde{T}$-indeed, we will often write $T \tilde{N}$ for $\tilde{T}$. We now replace $N$ by a nilpotent group $M$ in the genus of $N$; we will assume, as we may, that $T M=T N$ and $M_{p}=N_{p}$ for all primes $p$. We claim that $\operatorname{ker} \alpha$ is normal in $M$ under the natural embedding $\operatorname{ker} \alpha \subseteq T N=T M \subseteq M$. For $(\operatorname{ker} \alpha)_{p}$ is normal in $M_{p}$ for all primes $p$, which shows that $\operatorname{ker} \alpha$ is normal in $M$. We thus have a commutative diagram

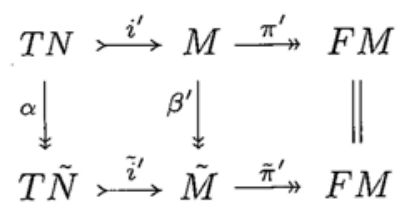

Theorem 1.1. The association $M \mapsto \tilde{M}$ defines a function $\alpha_{*}$ : $\mathfrak{G}(N) \rightarrow \mathfrak{G}(\tilde{N})$.

Proof: We have the commutative diagram (identifying $F M_{p}$ with $\left.F N_{p}\right)$

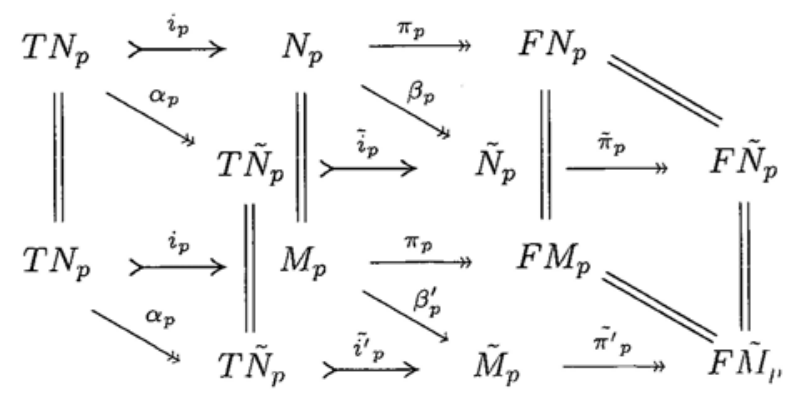

Now it is easy to prove that

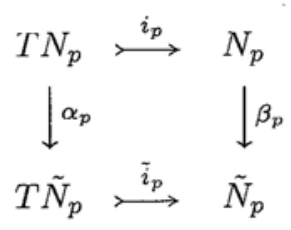

is also a push-out in the category of groups. Thus we have a (unique) homomorphism $\kappa: \tilde{N}_{p} \rightarrow \tilde{M}_{p}$ such that $\kappa \beta_{p}=\beta_{p}^{\prime}$ and $\kappa \tilde{i}_{p}=\tilde{i}_{p}^{\prime}$. We 
claim that $\tilde{\pi}_{p}^{\prime} \kappa=\tilde{\pi}_{p}$. For $\tilde{\pi}_{p}^{\prime} \kappa \beta_{p}=\tilde{\pi}_{p}^{\prime} \beta_{p}^{\prime}=\pi_{p}=\tilde{\pi}_{p} \beta_{p}$ and $\tilde{\pi}_{p}^{\prime} \kappa \tilde{i}_{p}=$ $\tilde{\pi}_{p}^{\prime} \tilde{i}_{p}^{\prime}=0=\tilde{\pi}_{p} \tilde{i}_{p}$. Thus the diagram

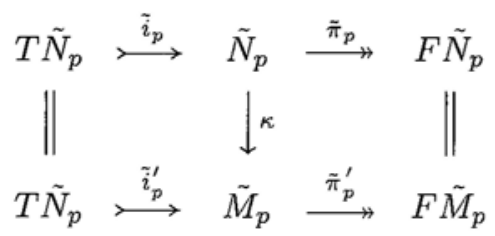

commutes, showing that $\kappa$ is an isomorphism. This proves that $\tilde{M} \in$ $\mathfrak{G}(\tilde{N})$ and establishes the theorem.

The following "functorial" properties of the association $\alpha \mapsto \alpha_{*}$ are obvious.

Theorem 1.2. (i) Id : TN $\rightarrow T N$ satisfies the condition $K$ and $\operatorname{Id}_{*}=\mathrm{Id}$.

(ii) If $\alpha: T N \rightarrow \tilde{T}=T \tilde{N}$ satisfies condition $K$ and $\tilde{\alpha}: T \tilde{N} \rightarrow \tilde{\tilde{T}}$ satisfies condition $K$, then $\tilde{\alpha} \alpha$ satisfies condition $K$ and $(\tilde{\alpha} \alpha)_{*}=\tilde{\alpha}_{*} \alpha_{*}$.

Proof: (i) is trivial. As to (ii), it suffices to remark that the existence of $\beta$ in (1.2) guarantees that $\alpha$ satisfies condition $K$. Thus we superimpose diagrams to produce

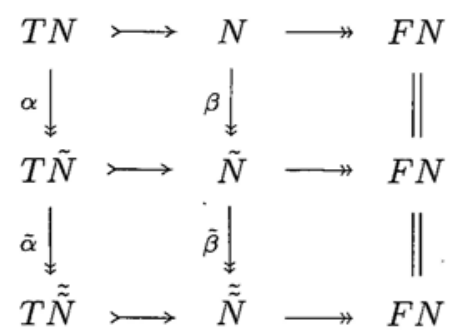

and deduce, first, that $\tilde{\alpha} \alpha$ satisfies condition $K$ and, second, that $(\tilde{\alpha} \alpha)_{*}=$ $\tilde{\alpha}_{*} \alpha_{*}$. For, just as (1.3) was derived in similar manner to (1.2) so 


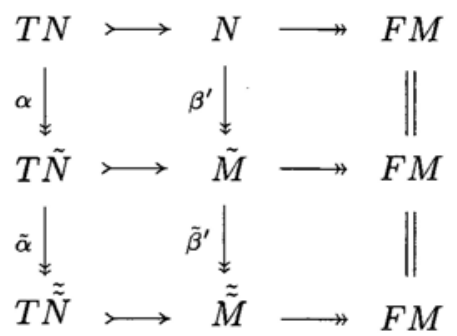

is derived in a similar manner to (1.4), and shows that

$$
\tilde{\tilde{M}}=\tilde{\alpha}_{*} \alpha_{*}(M)=(\tilde{\alpha} \alpha)_{*}(M) .
$$

We now make the further hypothesis that $N \in \mathfrak{R}_{0}$; this is equivalent to assuming that $F N$ is commutative. Since $F N=F \tilde{N}$ it follows that $\tilde{N} \in \mathfrak{R}_{0}$, so that both $\mathfrak{G}(N), \mathfrak{G}(\tilde{N})$ are finite abelian groups. (Notice that, in fact, $N \in \mathfrak{R}_{0}$ if and only if $\tilde{N} \in \mathfrak{R}_{0}$.) We then have

Theorem 1.3. Suppose that $N \in \mathfrak{R}_{0}$. Then $\alpha_{*}: \mathfrak{G}(N) \rightarrow \mathfrak{G}(\tilde{N})$ is a homomorphism.

Proof: Suppose that $K+L=M$ in $\mathfrak{G}(N)$. We continue to assume that

$$
T K=T L=T M=T N .
$$

Then, according to [HM], there exists an exhaustive pair $\varphi: N \rightarrow K$, $\psi: N \rightarrow L$, such that we may form the push-out (in $\mathfrak{R}$ )

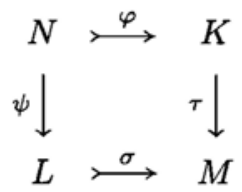

We recall from $[\mathbf{H M}]$ that an exhaustive pair $(\varphi, \psi)$ is defined by the requirements

(i) $\varphi$ or $\psi$ is a $T$-equivalence, where

$$
T=T(N)=\{p \mid N \text { has } p \text {-torsion }\} ;
$$

and (ii) for all primes $p, \varphi$ or $\psi$ is a $p$-equivalence. 
However, examination of the proof of Theorem 2.3 of $[\mathbf{H M}]$ shows that we may assume that both $\varphi$ and $\psi$ are T-equivalences. For having constructed $\varphi$ as a $T$-equivalence, we define

$$
P=\{p \mid \varphi \text { is not a } p \text {-equivalence }\}
$$

and then, modifying the argument in $[\mathbf{H M}]$, construct $\psi$ to be a $(P \cup T)$ equivalence.

With this strengthened sense of an exhaustive pair, we revert to (1.6). Then $\varphi, \psi$, when restricted to $T N$, are both isomorphisms, so we may suppose that both are identities on $T N$. We may then suppose that $\sigma$, $\tau$ are also identities on $T N$. Now let us factor out $\operatorname{ker} \alpha$ from each of $K$, $L, M, N$. Since $\operatorname{ker} \alpha \subseteq T N$, this gives rise to a commutative diagram

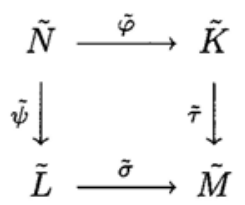

which is easily seen to inherit from (1.6) the property of being a push-out in $\Re$. Moreover, it is plain that $\tilde{\varphi}, \tilde{\psi}$ remain $T$-equivalences and that, for all primes $p, \tilde{\varphi}$ or $\tilde{\psi}$ is a $p$-equivalence. Since $T \tilde{N}$ is a quotient of $T N$ it is plain that $T(\tilde{N}) \subseteq T(N)$, so that $\tilde{\varphi}$ and $\tilde{\psi}$ are $T(\tilde{N})$-equivalences and $(\tilde{\varphi}, \tilde{\psi})$ is an exhaustive pair. We conclude that

$$
\tilde{K}+\tilde{L}=\tilde{M} \text { in } \mathfrak{G}(\tilde{N}),
$$

so that $\varphi$ is a homomorphism.

\section{A special case}

Since it has not yet proved possible to calculate $\mathfrak{G}(N)$ systematically for $N \in \Re_{0}$, it is not to be expected that we would have much success in trying to analyse the homomorphism $\alpha_{*}$ in the generality in which it has been introduced in the preceding section. However, we do find it possible to make some headway if we make the restrictive assumption that $\tilde{N} \in \mathfrak{R}_{1}$. We then prove

Theorem 2.1. Let $\alpha_{*}: \mathfrak{G}(N) \rightarrow \mathfrak{G}(\tilde{N})$ be defined as in Section 1 and let $\tilde{N} \in \mathfrak{R}_{1}$. Then $\alpha_{*}$ is a surjective homomorphism.

Proof: Since $\tilde{N} \in \mathfrak{R}_{0}$, it follows that $N \in \mathfrak{R}_{0}$ and $\alpha_{*}$ is a homomorphism. Now $\mathfrak{G}(\tilde{N})=0$ unless $F N$ is cyclic $[\mathbf{S}]$, [HS]. Thus, to avoid 
triviality, we assume $F N$ cyclic. Under this assumption, the top row of (1.2) splits for an action $\omega: F N \rightarrow \operatorname{Aut} T N$. Let $\sigma: F N \rightarrow N$ be a splitting $(\pi \sigma=1)$, so that, if $F N=\langle\xi\rangle$, then $\omega$ is given by

$$
\omega(\xi)(a)=y a y^{-1}, a \in T N, \text { where } y=\sigma(\xi) .
$$

We will often write $\xi \cdot a$ for $\omega(\xi)(a)$. We use $\beta \sigma: F N \hookrightarrow \tilde{N}$ to split the botton row of (1.2) and write $\tilde{\omega}: F N \rightarrow$ Aut $T \tilde{N}$ for the associated action. Note that $\tilde{\omega}$ is given by

$$
\tilde{\omega}(\xi)(\alpha a)=\alpha(\omega(\xi)(a)), \quad a \in T N .
$$

We write (2.2) more simply as

$$
\xi \cdot \alpha a=\alpha(\xi \cdot a), \quad a \in T N .
$$

Now let $\tilde{t}$ be the height of $\operatorname{ker} \tilde{\omega}$ in $F N$; that is, since $F N$ is cyclic, $\tilde{t}$ is the order of $\tilde{\omega}(\xi)$ in Aut $T \tilde{N}$. Then, by the main theorem of $[\mathbf{C H}]$,

$$
\mathfrak{G}(\tilde{N}) \cong(\mathbb{Z} / \tilde{t})^{*} /\{ \pm 1\}
$$

Moreover, we may choose the isomorphism (2.4) so that the group $\tilde{N}_{m}$, $m$ prime to $\tilde{t}$, corresponding to $[m] \in(\mathbb{Z} / \tilde{t})^{*} /\{ \pm 1\}$, is obtained from $\tilde{N}$ simply by replacing the action $\tilde{\omega}$ by a new action $\tilde{\omega}_{m}$, defined by

$$
\tilde{\omega}_{m}(\xi)(\tilde{a})=\tilde{\omega}\left(\xi^{m}\right)(\tilde{a}), \quad \tilde{a} \in T \tilde{N} .
$$

Of course we have freedom in (2.4) to choose $m$ within its given class $[m]$ without changing $\tilde{N}_{m}$. We will, in fact, choose $m$ to be a $T^{\prime}$-number, where $T=T(N)$ is the set of primes $p$ such that $N$ has $p$-torsion. To see that we can do this it suffices to notice that $m$ is prime to $\tilde{t}$ so that, by Dirichlet's Theorem, the residue class $[m]$ contains primes not in $T$.

With such a choice of $m$, we show that $\tilde{N}_{m}$ may be represented as $\alpha_{*}\left(N_{m}\right)$ for a suitable group $N_{m}$ in $\mathfrak{G}(N)$. We define $N_{m}$ to be the semi-direct product of $T N$ and $F N$ for the action $\omega_{m}: F N \rightarrow \operatorname{Aut} T N$, given by

$$
\omega_{m}(\xi)(a)=\omega\left(\xi^{m}\right)(a), \quad a \in T N .
$$

We first show that $N_{m} \in \mathfrak{G}(N)$. Consider the diagram

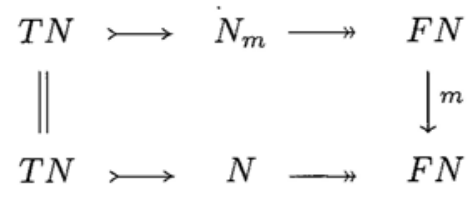


where the endomorphism of $F N$ is just $\xi \mapsto \xi^{m}$. Then (2.6) asserts that (2.7) satisfies the compatibility condition permitting us to complete it with $\varphi: N_{m} \rightarrow N$ to a commutative diagram. Now if $p \in T$ then $m$ : $F N \rightarrow F N$ is a $p$-equivalence, so that $\varphi: N_{m} \rightarrow N$ is a $p$-equivalence. If $p \notin T$ then $T N_{p}$ is the trivial group so both $N$ and $N_{m}$ are $p$-equivalent to $F N$ and hence $p$-equivalent to each other. Thus $N_{m} \in \mathfrak{G}(N)$.

Finally we show that $\alpha_{*}\left(N_{m}\right)=\tilde{N}_{m}$. Consider the diagrams
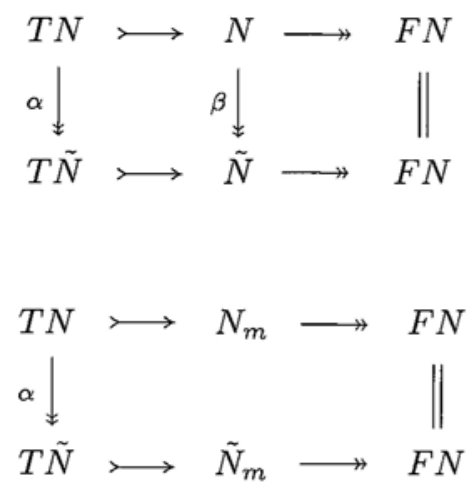

Recall that we are writing "." to indicate the actions of $F N$ on $T N$ or $T \tilde{N}$ in the first diagram; let us write "o" for the actions of $F N$ on $T N$ or $T \tilde{N}$ in the second diagram of (2.8). Then (2.3) $\xi \cdot \alpha a=\alpha(\xi \cdot a), a \in T N$ and (2.6) $\xi \circ a=\xi^{m} \cdot a, a \in T N$. Moreover, by (2.5), $\xi \circ \alpha a=\xi^{m} \cdot \alpha a$, $a \in T N$. But since $\xi \cdot \alpha a=\alpha(\xi \cdot a)$, it follows that $\xi^{m} \cdot \alpha a=\alpha\left(\xi^{m} \cdot a\right)$, whence

$$
\alpha(\xi \circ a)=\alpha\left(\xi^{m} \cdot a\right)=\xi^{m} \cdot \alpha a=\xi \circ \alpha a, \quad a \in T N .
$$

This, however, is precisely the compatibility condition guaranteeing the existence, in the second diagram of (2.8), of $\beta_{m}: N_{m} \rightarrow \tilde{N}_{m}$ making the diagram commutative. Then $\beta_{m}$ must be surjective. This, however, guarantees that

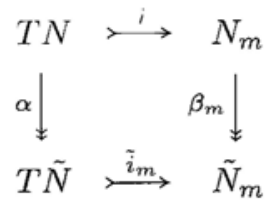

is a push-out in the category of groups and hence, by the uniqueness of push-outs, that $\tilde{N}_{m}=\alpha_{*}\left(N_{m}\right)$.

We now consider the groups $N_{m} \in \mathfrak{G}(N)$ constructed in the course of our proof of Theorem 2.1. We have immediately 
Corollary 2.2. Suppose $N_{m} \cong N_{m^{\prime}}$. Then $m \equiv m^{\prime} \bmod \tilde{t}$.

For if $N_{m} \cong N_{m^{\prime}}$ then $\tilde{N}_{m} \cong \tilde{N}_{m^{\prime}}$. We use Corollary 2.2 to obtain a non-cancellation result. We need some preliminary lemmas, the first of which addresses Remark 1 of [HM, Section 4].

Lemma 2.3. Let $N \in \Re_{0}$ and let $F Z N=n Z N$, where $Z N$ is the center of $N$ and $n=\exp T Z N$. Let $k$ be a $T$-number, where $T=T(N)$, and let $Q N=N / k F Z N$. Then $Q N$ is a finite group and $p \in T(Q N)$ if and only if $p \in T$.

Remark. In $[\mathbf{H M}]$ it was remarked that we achieved the same effect whether we defined $n$ to be the exponent or the order of $T Z N$; of course, in either case $F Z N$ is free abelian.

Proof of Lemma 2.3: Since $[N, N]$ is finite and $N$ is $f g$ nilpotent, $N / Z N$ is finite. Also $Z N$ is $f g$ so $Z N / k n Z N$ is finite. Hence $N / k n Z N$ is finite. Now let $Z N=F \oplus T Z N$, with $F f g$ free abelian. Then $k F Z N=k n F$, so

$$
Z N / k F Z N=F / k n F \oplus T Z N .
$$

Also we have an exact sequence

$$
Z N / k F Z N \longmapsto Q N \rightarrow N / Z N .
$$

From (2.9) we infer, for an arbitrary prime $p$,

$Z N$ has $p$-torsion $\Rightarrow Z N / k F Z N$ has $p$-torsion $\Rightarrow N$ has $p$-torsion.

Thus, from (2.10),

$Q N$ has $p$-torsion $\Rightarrow Z N / k F Z N$ or $N / Z N$ has $p$-torsion $\Rightarrow N$ has $p$-torsion; and $N$ has $p$-torsion $\Rightarrow Z N$ or $N / Z N$ has $p$-torsion $\Rightarrow Z N / k F Z N$ or $N / Z N$ has $p$-torsion $\Rightarrow Q N$ has $p$-torsion.

This completes the proof.

Lemma 2.4. Let $N \in \Re_{0}$ with $F N$ cyclic, $F N=\langle\xi\rangle$. Let $t$ be the order of $\omega(\xi) \in$ Aut $T N$. Then $t$ is a $T$-number, where $T=T(N)$.

Proof: Certainly $F Z N$ is a free cyclic group. Suppose it is generated by $\left(a, \xi^{s}\right), a \in T N$. By conjugating with $(1, \xi)$ it is clear that $\xi \cdot a=a$. Let $k$ be the order of $a$. Then $\left(a, \xi^{s}\right)^{k}=\left(1, \xi^{s k}\right)$. Now, since $t$ is the order of $\omega(\xi)$, we infer that $t \mid s k$. 
We compute $Q N$ as in Lemma 2.3. We have

$$
N=\langle T N, y\rangle, \text { where } y=(1, \xi)
$$

$k F Z N=\left\langle y^{s k}\right\rangle$ (we confuse additive and multiplicative notation here!)

Thus, $Q N=\left\langle T N, y \mid y^{s k}=1\right\rangle$.

When we abelianize $Q N$ we get generators from $(T N)_{a b}$, together with $\bar{y}$; and the only relation involving $\bar{y}$ is $\bar{y}^{s k}=1$. Thus $s k \mid \exp Q N_{a b}$, whence $t \mid \exp Q N_{a b}$. Now since $Q N$ is a finite nilpotent group, $T(Q N)=$ $T\left(Q N_{a b}\right)$, so that, by Lemma 2.3 ,

$$
T=T(N)=T\left(Q N_{a b}\right) .
$$

Since $t \mid \exp Q N_{a b}, t$ is a $T\left(Q N_{a b}\right)$-number. Hence, by (2.11), $t$ is a $T$ number.

Before stating our non-cancellation result, we observe that the invariant $t$ provides us with a partial converse to Corollary 2.2. Thus we may prove

Theorem 2.5. (i) $\tilde{t} \mid t$; (ii) if $m \equiv m^{\prime} \bmod t$, then $N_{m} \cong N_{m^{\prime}}$.

Proof: (i) follows immediately from (2.3) and the fact that $\alpha$ is surjective.

As to (ii), observe first that $N_{m} \cong N_{-m}$; for we have the diagram

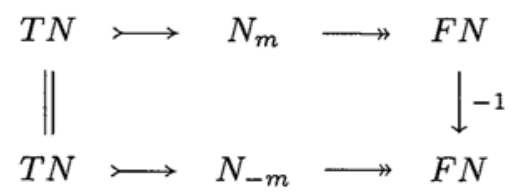

satisfying the obvious compatibility condition, giving rise to an isomorphism $N_{m} \cong N_{-m}$. Further we have an actual equality between $N_{m}$ and $N_{m+q t}$ since $\xi^{m+q t} \cdot a=\xi^{m} \cdot a$, for all $a \in T N$.

We are now ready to enunciate our non-cancellation theorem; recall that we have constructed a group $N_{m}$ in $\mathfrak{G}(N)$ for each $m$ such that $m$ is a $T^{\prime}$-number prime to $\tilde{t}$; and that $N_{m} \cong N_{m^{\prime}} \Rightarrow m \equiv \pm m^{\prime} \bmod \tilde{t}$.

Theorem 2.6. $N_{m} \times C \cong N \times C$, where $C$ is cyclic infinite.

Proof: Since $m$ is a $T^{\prime}$-number it follows from Lemma 2.4 that $m$ is prime to $t$, the order of $\omega(\xi)$ in Aut $T N$. Let $A=\left(\begin{array}{cc}m & t \\ r & s\end{array}\right)$ be a unimodular matrix over $\mathbb{Z}$; let $C=\langle\eta\rangle$ and interpret $A$ as the automorphism of $F N \times C$ given by $\xi \mapsto \xi^{m} \eta^{r}, \eta \mapsto \xi^{t} \eta^{s}$. Consider the diagram 


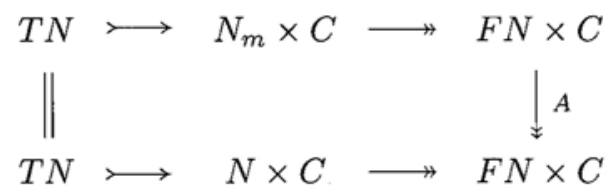

We claim that (2.12) satisfies the compatibility condition. For $C$ operates trivially on $T N$ so we may write, for the top row of (2.12),

$$
\xi \circ a=\xi^{m} \cdot a, \quad \eta \circ a=a, \quad a \in T N .
$$

and, for the bottom row of $(2.12)$,

$$
\eta \cdot a=a, \quad a \in T N .
$$

Moreover, each of $N_{m} \times C, N \times C$ is the semi-direct product for the given actions. Further

$$
\begin{aligned}
& A \xi \cdot a=\xi^{m} \eta^{r} \cdot a=\xi^{m} \cdot a=\xi \circ a, \\
& A \eta \cdot a=\xi^{t} \eta^{s} \cdot a=a=\eta \circ a,
\end{aligned}
$$

by (2.13) and (2.14). It follows that we may find

$$
\varphi: N_{m} \times C \longmapsto N \times C
$$

completing (2.12) to a commutative diagram. It is then clear that $\varphi$ is an isomorphism.

Now to obtain an actual non-cancellation example, it suffices to find an example of the data of Theorem 2.1 in which $\tilde{t} \not \equiv 1,2,3,4,6$. In the next section we show, in fact, how to construct examples with any given $\tilde{t}$.

\section{Examples}

We may apply Theorem 1.1 by factoring $[T N, T N],[N, N] \cap T N, T Z N$, $Z N \cap T N$ out of $T N$ and $N$ and letting $\alpha, \beta$ be the associated quotient maps. The first is especially interesting for then $T \tilde{N}$ is commutative, but $\tilde{N}$, in general, is not. If $N \in \Re_{0}$, we may apply Theorem 1.3; and we may further hope that $\tilde{N} \in \Re_{1}$ so that we can apply Theorem 2.1. If $F N$ is cyclic we will only need to verify condition (iii) for membership of $\Re_{1}$ (see the Introduction), and, if $T \tilde{N}$ is also cyclic, condition (iii) is automatically verified. 
We now give an example (or, rather, a family of examples) which gives rise to a group $\tilde{N}$ in $\Re_{1}$ (although $T \tilde{N}$ is not cyclic), and thus to the construction of non-trivial genera $\mathfrak{G}(N)$ for groups $N$ in $\mathfrak{R}_{0}$, with $T N$ non-commutative, and to explicit non-cancellation results, based on Corollary 2.2 and Theorem 2.6.

Given $\tilde{t}$, choose $n$ and $u$ such that (i) $n$ is even; (ii) $p|n \Rightarrow p| u-1$, for all primes $p$; (iii) the order of $u \bmod n$ is $\tilde{t}$. Notice that (i) and (ii) imply that $u$ is odd. As examples of possible choices for $n$ and $u$, we have:

It $\tilde{t}$ is odd, say $\tilde{t}=p_{1}^{\ell_{1}} p_{2}^{\ell_{2}} \ldots p_{\lambda}^{\ell_{\lambda}}$, choose

$$
n=2 p_{1}^{\ell_{1}+1} p_{2}^{\ell_{2}+1} \ldots p_{\lambda}^{\ell_{\lambda}+1}, \quad u=1+2 p_{1} p_{2} \ldots p_{\lambda} ;
$$

if $\tilde{t}$ is even, say $\tilde{t}=2^{\ell_{1}} p_{2}^{\ell_{2}} \ldots p_{\lambda}^{\ell_{\lambda}}$, choose

$$
n=2^{\ell_{1}+2} p_{2}^{\ell_{2}+1} \ldots p_{\lambda}^{\ell_{\lambda}+1}, \quad u=1+4 p_{2} \ldots p_{\lambda} .
$$

Now set $T N=\langle x, y, z| x^{2}=y^{2}=z^{2 n}=1,[x, y]=z^{n},[x, z]=[y, z]=$ 1). Obviously $T N$ is nilpotent of class 2. Let $F N=\langle\xi\rangle$ operate on $T N$ by the rule

$$
\xi \cdot x=x, \quad \xi \cdot y=y, \quad \xi \cdot z=z^{u} .
$$

This clearly describes an automorphism of $T N$ since $u$ is prime to $n$ by (ii) above and hence, being odd, prime to $2 n$. Moreover, $z^{u n}=z^{n}$, again because $u$ is odd.

We claim that the action (3.1) is nilpotent. For we have $\Gamma_{F N}^{0} T N=$ $T N$,

$$
\begin{aligned}
& \Gamma_{F N}^{1} T N=\left\langle z^{u-1}, z^{n}\right\rangle, \\
& \Gamma_{F N}^{2} T N=\left\langle z^{(u-1)^{2}}, z^{(u-1) n}\right\rangle=\left\langle\left(z^{(u-1)^{2}}\right\rangle,\right. \\
& \Gamma_{F N}^{3} T N=\left\langle z^{(u-1)^{3}}\right\rangle, \ldots,
\end{aligned}
$$

and thus, again by (ii) above, $\Gamma_{F N}^{k} T N=\{1\}$ for $k$ sufficiently large. If, then, we form the semi-direct product $N$ of $T N$ and $F N$ for this action, $N$ is a nilpotent group and, indeed, $N \in \mathfrak{R}_{0}$.

Now $[T N, T N]=\left\langle z^{n}\right\rangle$. Thus we may factor out $[T N, T N]$ to form

$$
\tilde{T}=(T N)_{a b}=\langle\tilde{x}, \tilde{y}, \tilde{z} \mid 2 \tilde{x}=2 \tilde{y}=n \tilde{z}=0\rangle,
$$

and, following the procedure of Section 1, we have the commutative diagram 


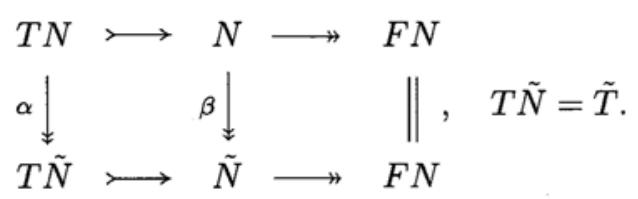

Now $F N$ acts on $T \tilde{N}$ by

$$
\xi \cdot \tilde{x}=\tilde{x}, \quad \xi \cdot \tilde{y}=\tilde{y}, \quad \xi \cdot \tilde{z}=u \tilde{z},
$$

so that

$$
\xi \cdot \tilde{a}=u \tilde{a}, \text { for all } \tilde{a} \in T \tilde{N} .
$$

Moreover, $\exp T \tilde{N}=n$, so that $\tilde{N} \in \Re_{1}$ by (3.5) and

$$
\mathfrak{G}(\tilde{N}) \cong(\mathbb{Z} / \tilde{t})^{*} /\{ \pm 1\}
$$

by condition (iii). Thus

$$
\alpha_{*}: \mathfrak{G}(N) \rightarrow(\mathbb{Z} / \tilde{t})^{*} /\{ \pm 1\}
$$

and $\mathfrak{G}(N)$ is a non-trivial group, provided that $\tilde{t} \neq 1,2,3,4,6$.

Now $u^{\tilde{t}} \equiv 1 \bmod n$. Thus $u^{2 \tilde{t}} \equiv 1 \bmod 2 n$, so that $t=2 \tilde{t}$ or $\tilde{t}$. Moreover, we may follow the procedure of Section 2 to construct $N_{m}$ if $m$ is prime to $\tilde{t}$ and a $T^{\prime}$-number, where $T=T(N)$. Plainly $\exp T N=2 n$, so $T$ consists of the prime divisors of $n$.

Let us now insist, for simplicity, as we clearly may, that $\tilde{t}$ and $n$ have precisely the same prime divisors, except that $2 \mid n$ even if $\tilde{t}$ is odd. Thus we can construct $N_{m}$ if $m$ is prime to $\tilde{t}$, with the additional condition that $m$ is odd, even if $\tilde{t}$ is odd. We thus have

Theorem 3.1. For a given $\tilde{t}$, choose $(n, u)$ as above and construct the group $N$ as described. Then there is a surjective homomorphism

$$
\alpha_{*}: \mathfrak{G}(N) \rightarrow(\mathbb{Z} / \tilde{t})^{*} /\{ \pm 1\} .
$$

We may also construct $N_{m} \in \mathfrak{G}(N)$ for any odd $m$ prime to $\tilde{t}$, and

$$
m \equiv \pm m^{\prime} \bmod 2 \tilde{t} \Rightarrow N_{m} \cong N_{m^{\prime}} \Rightarrow m \equiv \pm m^{\prime} \bmod \tilde{t} .
$$

Moreover, $N_{m} \times C \cong N \times C$ for any odd $m$ prime to $\tilde{t}$.

Finally, we become even more specific! Let $\tilde{t}$ itself be odd and choose $(n, u)$ as follows (this modifies slightly our earlier example of a possible choice). Thus, if $\tilde{t}=p_{1}^{\ell_{1}} p_{2}^{\ell_{2}} \ldots p_{\lambda}^{\ell_{\lambda}}$, choose

$$
n=2 p_{1}^{\ell_{1}+1} p_{2}^{\ell_{2}+1} \ldots p_{\lambda}^{\ell_{\lambda}+1}, \quad u=1+4 p_{1} p_{2} \ldots p_{\lambda} .
$$


The effect of this choice is that $t=\tilde{t}$, since the order of $u \bmod 2 n$ is the same (i.e., $\tilde{t}$ ) as the order of $u \bmod n$. Thus, with the choice (3.9) -of course, other choices may have the same effect- we may improve (3.8) to

$$
m \equiv \pm m^{\prime} \bmod \tilde{t} \Leftrightarrow N_{m} \cong N_{m^{\prime}} .
$$

Example 3.1. Let $\tilde{t}=35$. Then, according to (3.9), we choose $n=2450, u=141$. Now $(\mathbb{Z} / 35)^{*} /\{ \pm 1\} \cong C_{12}$, its elements being [2], [4], [8], [16], [32], [29], [23], [11], [22], [9], [18], [1]. Thus, since we must take $m$ odd, we have, as possible values of $m$,

$$
m=33,31,27,19,3,29,23,11,13,9,17,1 .
$$

Each of these values of $m$ yields, according to (3.8'), a group $N_{m}$ in $\mathfrak{G}(N)$, no two of which are isomorphic. On the other hand all the groups $N_{m} \times C$, as $m$ runs through the values of (3.10), are isomorphic.

Remark. It is easy to extend Theorem 2.1 to the study of $\mathfrak{G}\left(N^{k}\right)$, $k \geq 2$, where $N^{k}$ is the direct product of $k$ copies of $N$. For we recall from $[\mathbf{C H}]$ the surjective homomorphism $\rho: \mathfrak{G}(N) \rightarrow \mathfrak{G}\left(N^{k}\right), N \in \mathcal{N}_{0}$, given by $\rho(M)=M \times N^{k-1}$. Plainly we have a commutative diagram

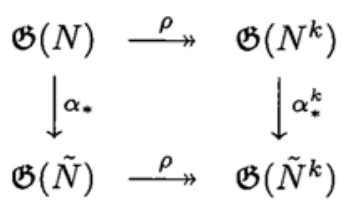

so that, since $\alpha_{*}$ is surjective, so is $\alpha_{*}^{k}$. Since we have calculated $\mathfrak{G}\left(\tilde{N}^{k}\right)$ for $\tilde{N} \in \mathfrak{R}_{1}[\mathbf{S}]$, [HS2], we may extend the applications in this section from $\mathfrak{G}(N)$ to $\mathfrak{G}\left(N^{k}\right)$. We leave the details to the reader.

\section{References}

[CH] Casacuberta C. AND Hilton P., Calculating the Mislin genus for a certain family of nilpotent groups, Comm. in Algebra 19(7) (1991), 2051-2069.

[H1] HiLton P., Localization of crossed modules, Springer Lecture Notes in Mathematics 1029, 1984, pp. 311-324.

[H2] Hilton P., On the genus of nilpotent groups and spaces, Israel Journ. Math. 54(1) (1986), 1-13. 
[H3] Hilton P., On the extended genus, Acta Math. Sinica 4(4) (1988), 372-382.

[HM] Hilton P. AND MisLin G., On the genus of a nilpotent group with finite commutator subgroup, Math. Zeit. 146 (1976), 201-211.

[HS1] Hilton P. AND SchucK C., On the structure of nilpotent groups of a certain type, to appear.

[HS2] Hilton P. AND SCHUCK C., Calculating the genus of a certain nilpotent group, Bulletin de la Sociedad Matemática Mexicana, to appear.

[M] Mislin G., Nilpotent groups with finite commutator subgroups, Springer Lecture Notes in Mathematics 418, 1974, pp. 103-120.

[S] SCHUCK C., Some contributions to the study of a class of nilpotent groups, Ph. D. Dissertation, SUNY Binghamton, 1992.

Department of Mathematical Sciences

SUNY Binghamton

Binghamton, New York, 13902-6000

U.S.A.

Primera versió rebuda el 10 de Novembre de 1993, darrera versió rebuda el 14 de Març de 1994 